Open Access Full Text Article

REVIEW

\title{
Is it possible to prevent chemotherapy-induced heart failure with cardiovascular drugs - the review of the current clinical evidence
}

This article was published in the following Dove Press journal:

Therapeutics and Clinical Risk Management

\section{Katarzyna Korzeniowska (ID Jerzy Jankowski (D) \\ Artur Cieślewicz (D) \\ Anna Jabłecka}

Department of Clinical Pharmacology, Poznan University of Medical Sciences, Poznan 6I-848, Poland
Correspondence: Artur Cieślewicz Department of Clinical Pharmacology, Poznan University of Medical Sciences, Długa I/2 Str., Poznan 61-848, Poland

Tel +48 6I 8549216

$\mathrm{Fax}+48618533161$

Email artcies@ump.edu.pl

\begin{abstract}
Cardiovascular diseases and cancer are the most common death causes in the USA and Europe. Moreover, many patients suffer from both of these conditions - a situation which may result from cardiotoxicity of anticancer treatment. In order to reduce the severity of this adverse effect, various methods have been proposed, including the usage of new drug forms and less toxic analogs, omitting the combinations of potentially cardiotoxic drugs and introducing potential cardioprotective agents to the therapy. However, prevention of cardiotoxicity still seems to be insufficient. The article reviews the results of current studies on the use of cardiovascular drugs in the prevention of cardiotoxicity. Based on this knowledge, the most promising cardioprotective drugs seem to be carvedilol, nebivolol, enalapril, and candesartan, as they prevent heart remodeling and correct elevated resting heart rate, which directly affects mortality. Alternatively, in case of adverse reactions, statins might be considered.
\end{abstract}

Keywords: chemotherapy, cardiotoxicity, heart failure, prevention

\section{Introduction}

Cardiovascular diseases and cancer are the most common death causes in the USA and Europe, although long-term survival of oncological patients has increased in the last years, reaching $64 \%$ of 5 -year survival rate, $41 \%$ of 10 -year survival rate and $15 \%$ of 20 -year survival rate. Moreover, many patients suffer from both of these conditions - a situation which may result from cardiotoxicity of anticancer treatment. $^{1,2}$ The most frequent symptom of such cardiotoxicity is LVEF (left ventricular ejection fraction) reduction which may indicate the development of left ventricular dysfunction and lead to congestive heart failure. Other cardiotoxicity symptoms include arrhythmias, changes in blood pressure or cardiomyopathy. Data from United Network for Organ Sharing (UNOS) and Interagency Registry for Mechanically Assisted Circulatory Support (INTERMACS) indicate that $0.5 \%$ to $2.5 \%$ patients with left ventricular assist devices and orthotopic heart transplantation had cancer treatment-related cardiomyopathy. ${ }^{3,4}$

To address the problem of cardiotoxicity, various methods have been introduced, including the application of high-sensitivity diagnostic methods, the use of new forms and less toxic analogs of chemotherapeutics, and coadministration of dexrazoxane..$^{5-7}$ Nevertheless, the use of cardiovascular drugs for cardioprotection of oncological patients has not been recommended so far." ${ }^{8,9}$ Only the "2016 
European Guidelines on cardiovascular disease prevention in clinical practice" stated that early preventive treatment should be applied to achieve maximum efficacy in counteracting anthracycline-induced cardiotoxicity in high-risk patients. ${ }^{10}$ Cardiovascular drugs have been studied for many years as potential agents preventing cardiotoxicity of oncological treatment. ${ }^{11-13}$ Therefore, the subject of the presented article is to review the results of clinical trials assessing the cardioprotection of patients undergoing oncological treatment.

\section{Methods}

PubMed database was searched using the following terms: cardiotoxicity, prevention, RAA system drugs, $\beta$-blockers, trimetazidine, ACEI, ARB, statin, ivabradine, coenzyme Q10, ranolazine, aldosterone antagonist.

\section{$\beta$-blockers}

\section{Nebivolol}

Cardioprotective effect of nebivolol was confirmed in an experimental model of doxorubicin-induced cardiac toxicity by Imbaby et al. They have found that it improved survival rate, ECG (electrocardiogram) parameters, cardiac enzymes, oxidative stress, apoptosis, and histopathological picture which are potentiated by adding a low dose of curcumin. ${ }^{14}$ The protective effect of $5 \mathrm{mg}$ daily dose of nebivolol in breast cancer patients receiving anthracyclinebased chemotherapy was also assessed in the randomized, double-blind, placebo-controlled clinical study by Kaya et al (Table 1). At 6-month after chemotherapy, echocardiographic measurements of left ventricular end-systolic diameters (LVESD) and left ventricular end-diastolic diameters (LVEDD) remained unchanged in the nebivolol group (LVESD: $30.4 \pm 3.5$ to $31.0 \pm 3.6 \mathrm{~mm}, p=0.20$; LVEDD: $47.0 \pm 4.4$ to $47.1 \pm 4.0 \mathrm{~mm}, p=0.93$ ) in contrast to significant increase in the control group. The placebo group had also lower LVEF (left ventricular ejection fraction) than the nebivolol group $(57.5 \pm 5.6 \%$ vs $63.8 \pm 3.9 \%$, $p=0.01$ ). The level of N-terminal pro-brain natriuretic peptide (NT-pro-BNP) remained static in the nebivolol group while it significantly increased in the placebo group. ${ }^{15}$

\section{Carvedilol}

In the first clinical randomized trial with carvedilol $(12,5$ mg once-daily), performed by Kalay et al (Table 1), it was found that 6-month treatment maintained LVESD, LVEDD, and systolic function compared with placebo.
The interventions were initiated prior to the start of chemotherapy and continued for six months. ${ }^{16}$ Similar results were obtained by Elitok et al (Table 1) in a group of female patients using the same dose of the drug. The authors have found that septal and lateral systolic strain and strain rate values were significantly lower in control patients compared to the carvedilol group. ${ }^{17}$

Zamani et al (Table 1) carried out a randomized, controlled trial where patients with lymphoma or breast cancer were administered carvedilol. They observed that a lower dose of carvedilol (6,25 mg/day) did not have a significant effect on the prevention of diastolic and systolic dysfunction, contrary to the dose $12.5 \mathrm{mg} /$ day. These results suggested that the cardioprotective effect was dosedependent. ${ }^{18}$

Recently published CECCY trial (Table 1) was conducted on 192 patients with breast cancer but without cardiovascular diseases, receiving cyclophosphamide, doxorubicin, and paclitaxel. It was found that carvedilol, initially with a dose of $3.125 \mathrm{mg}$ twice a day to a maximum dose of $25 \mathrm{mg}$ every $12 \mathrm{hrs}$, affected only troponin I, B-type natriuretic peptide, and diastolic dysfunction. The study did not find any significant effect of carvedilol on LVEF. Symptomatic hypotension was reported in 3 patients in the carvedilol group; one of them was withdrawn from the study. ${ }^{19}$

A meta-analysis of 8 randomized controlled trials carried out by Kheiri et al (Table 1), assessing changes in ejection fraction of patients treated with anthracyclines, revealed that carvedilol protected the patients from LVEF reduction (mean differences between carvedilol and placebo groups: $2.41 \%$ ). The authors concluded that prophylactic use of carvedilol might have a cardioprotective effect, reducing the early onset of left ventricular dysfunction. ${ }^{20}$

\section{Bisoprolol}

During the MANTICORE 101-Breast trial (Table 1), it was found that bisoprolol administered to patients treated with trastuzumab (who received a non-anthracyclinebased chemotherapy), titrated weekly to $10 \mathrm{mg}$, attenuated trastuzumab-mediated declines in LVEF but did not prevent ventricular remodeling. No significant adverse effects were noted during the study; lower heart rate was observed after completed chemotherapy. ${ }^{21}$

\section{Metoprolol}

Georgakopoulos et al (Table 1), in a randomized control trial with long (36 months) follow-up observed decreased 


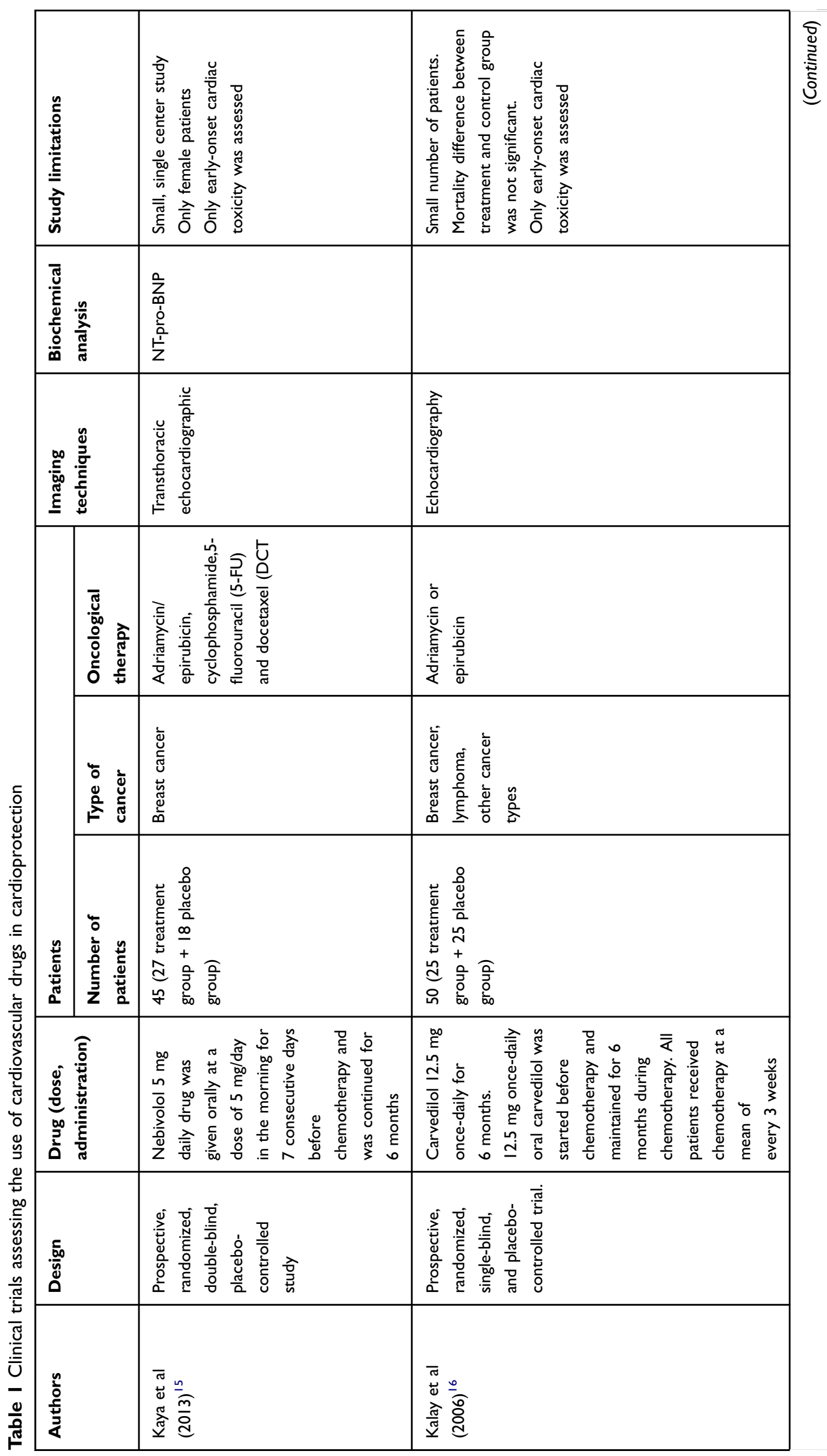




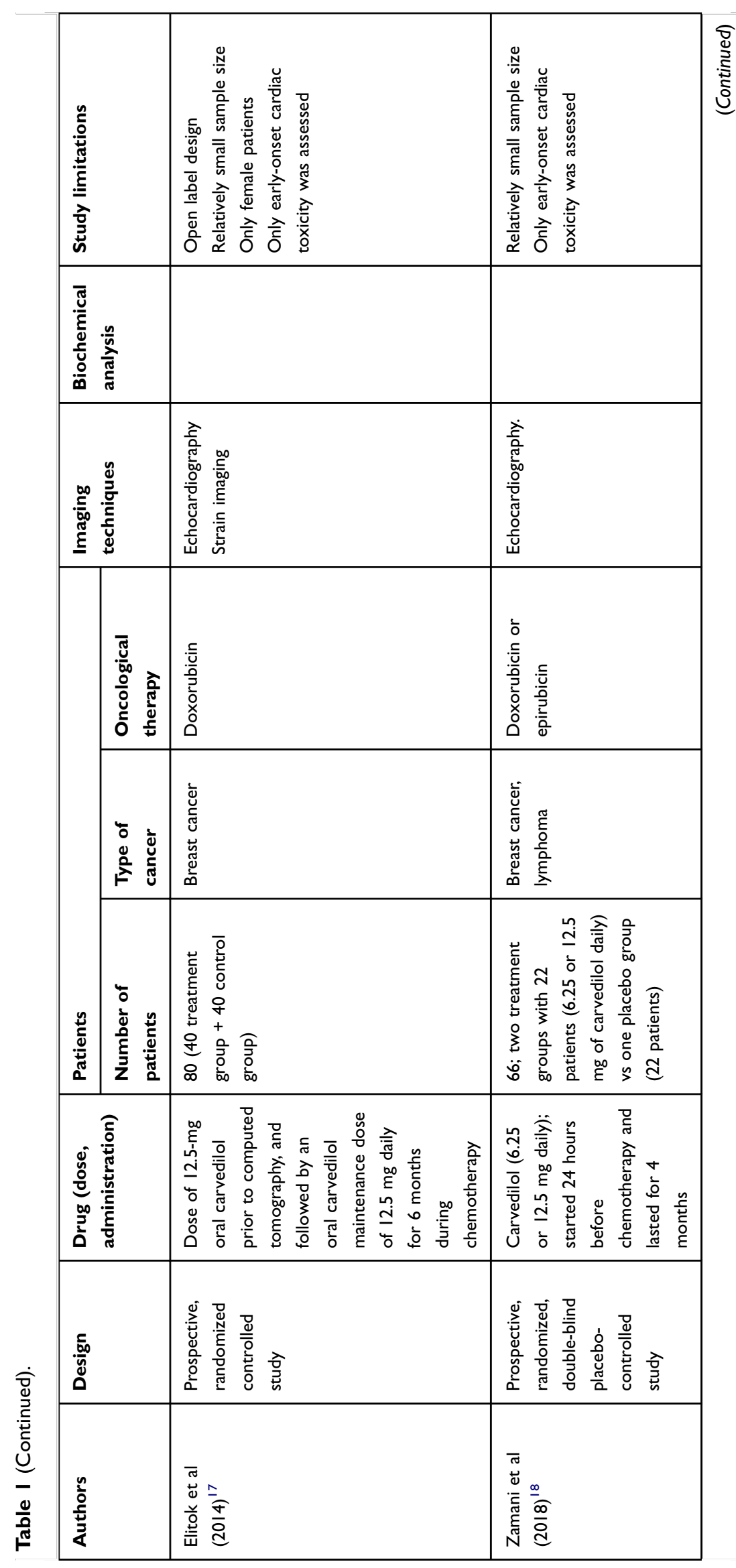




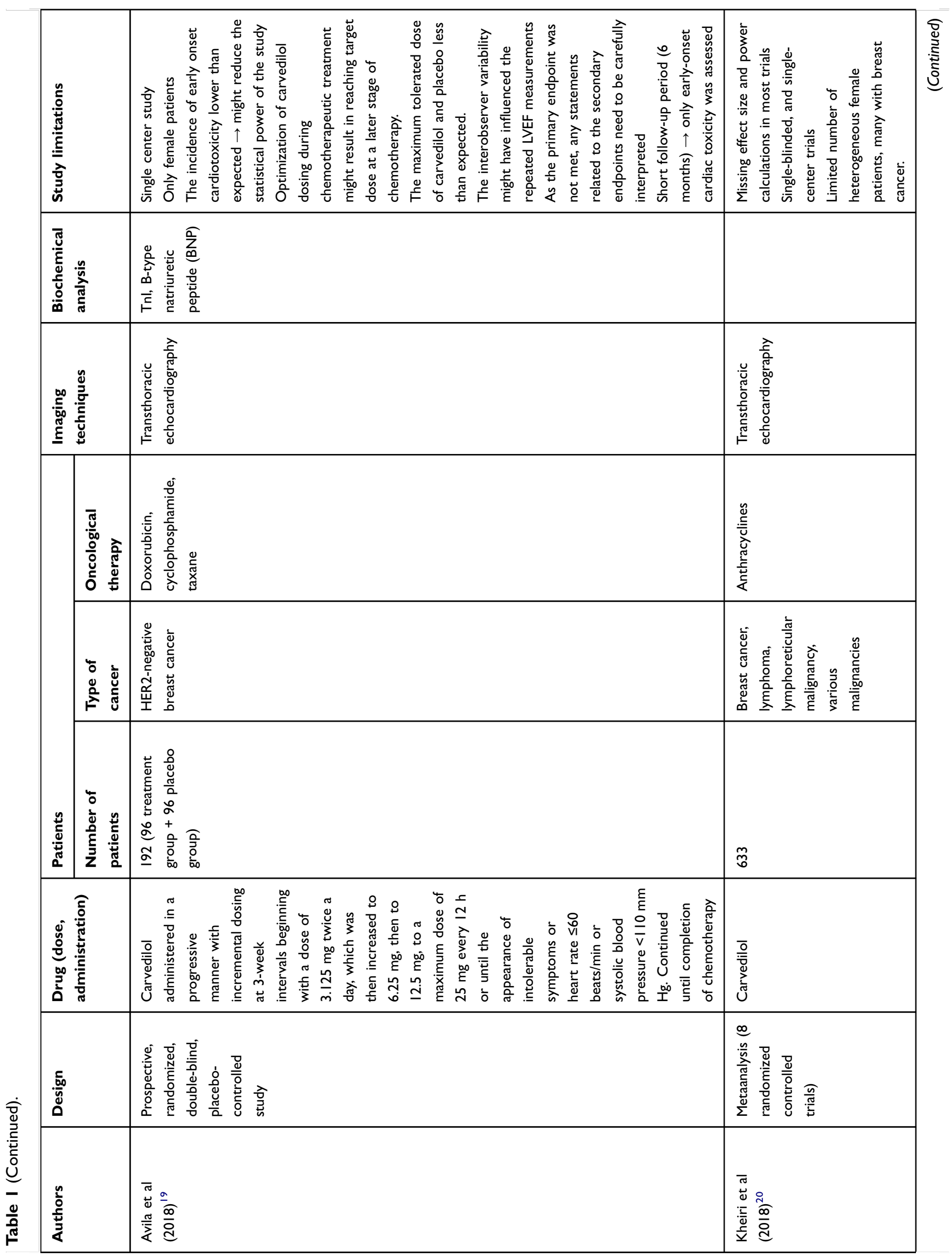




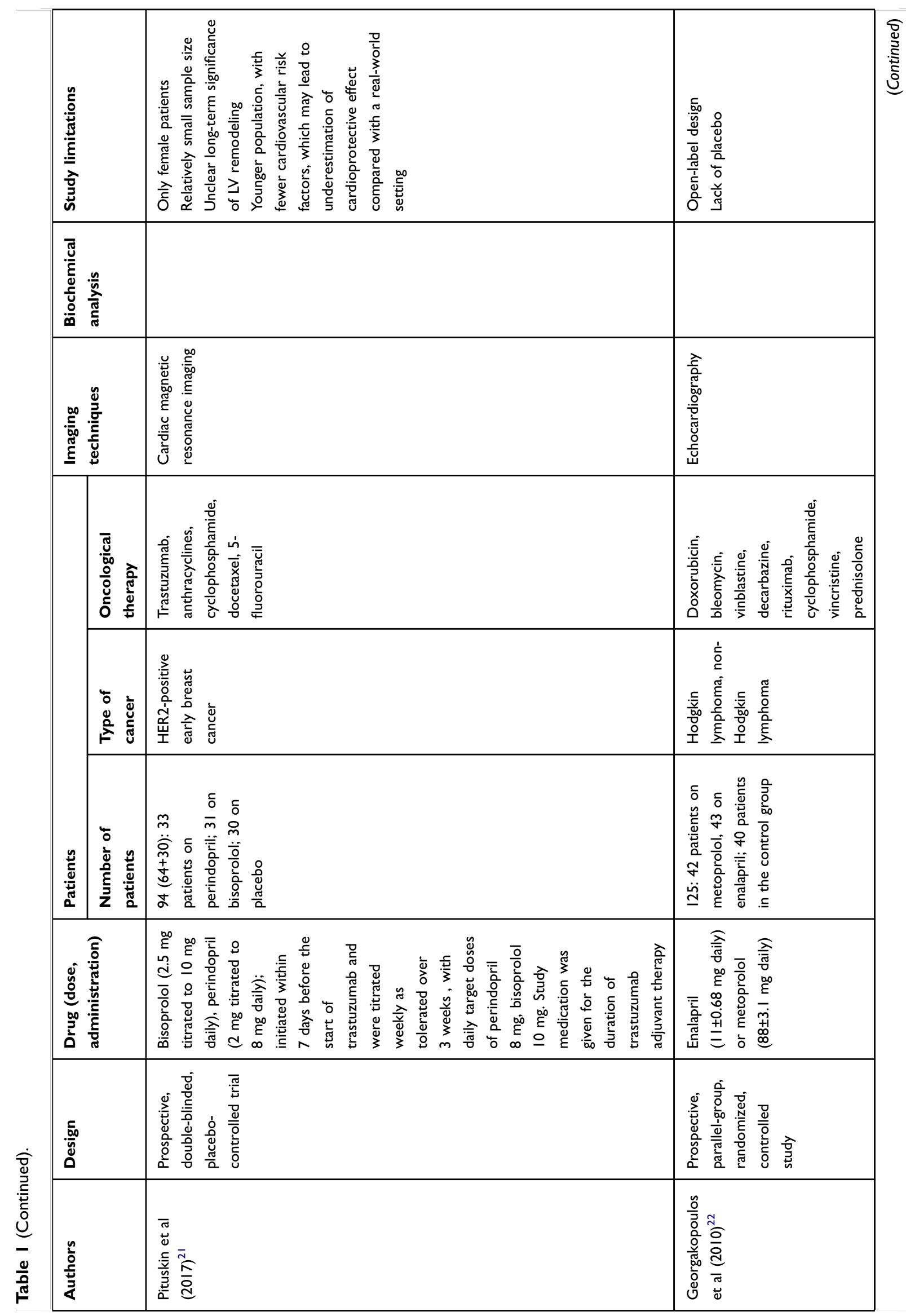




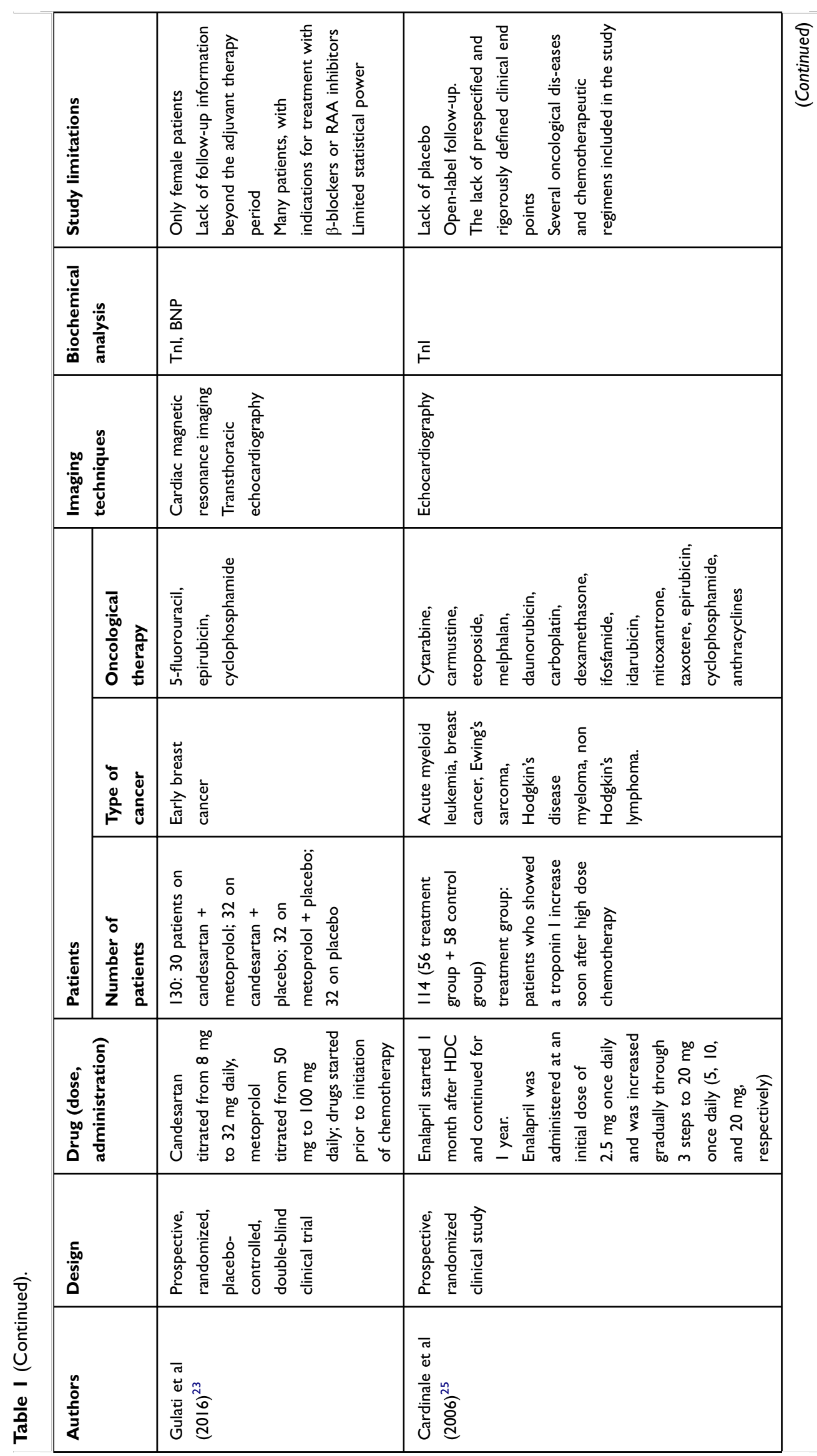




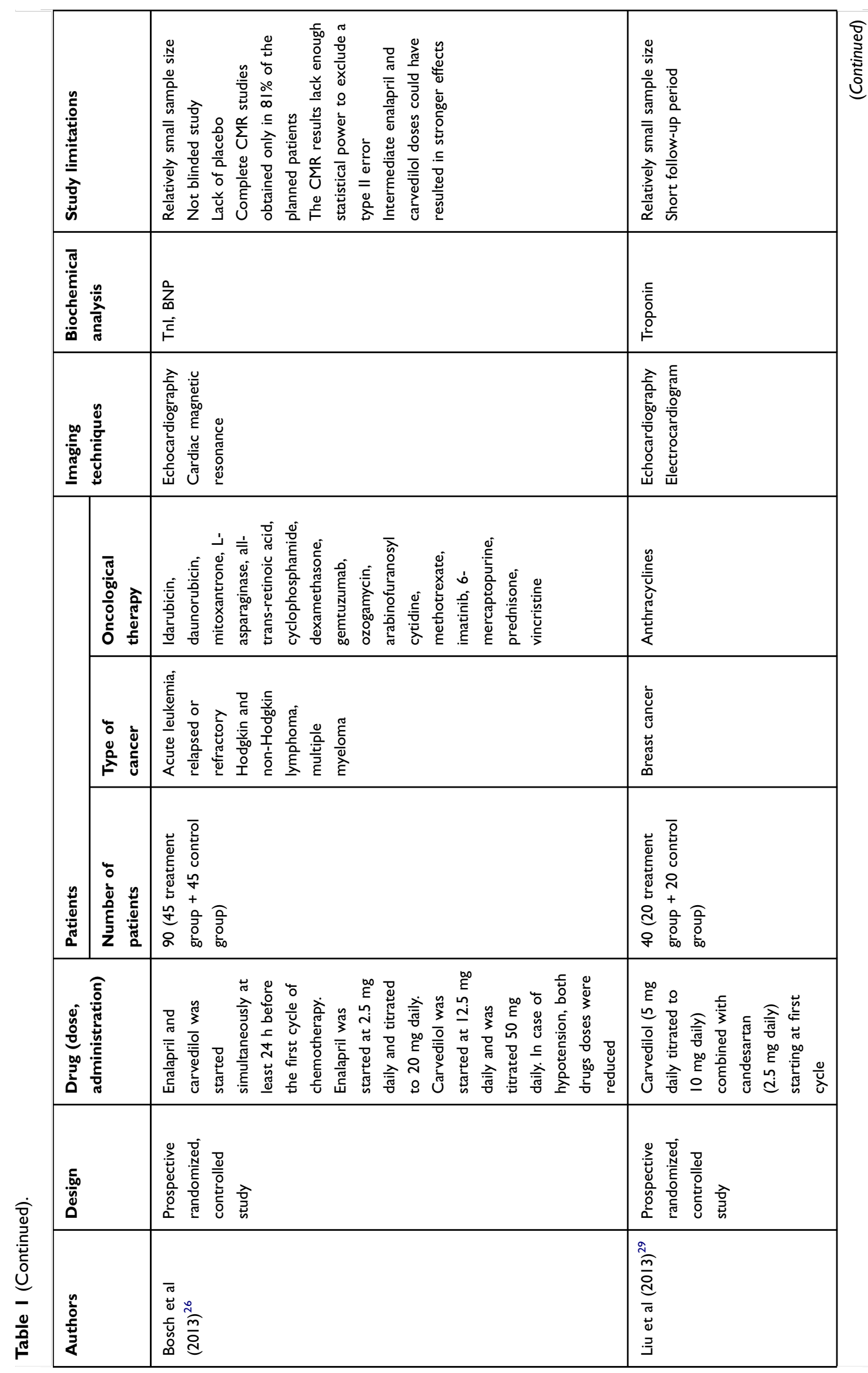




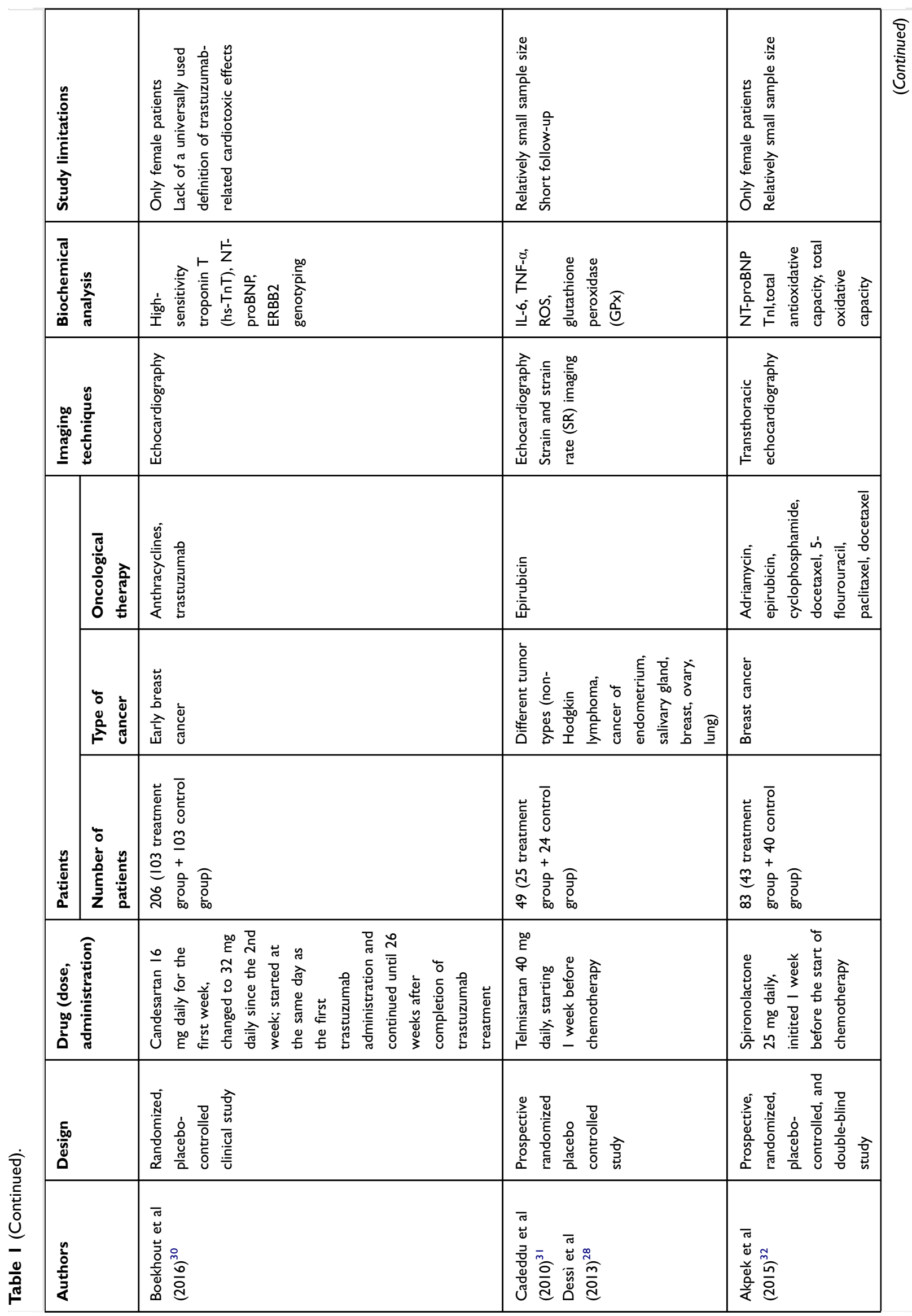




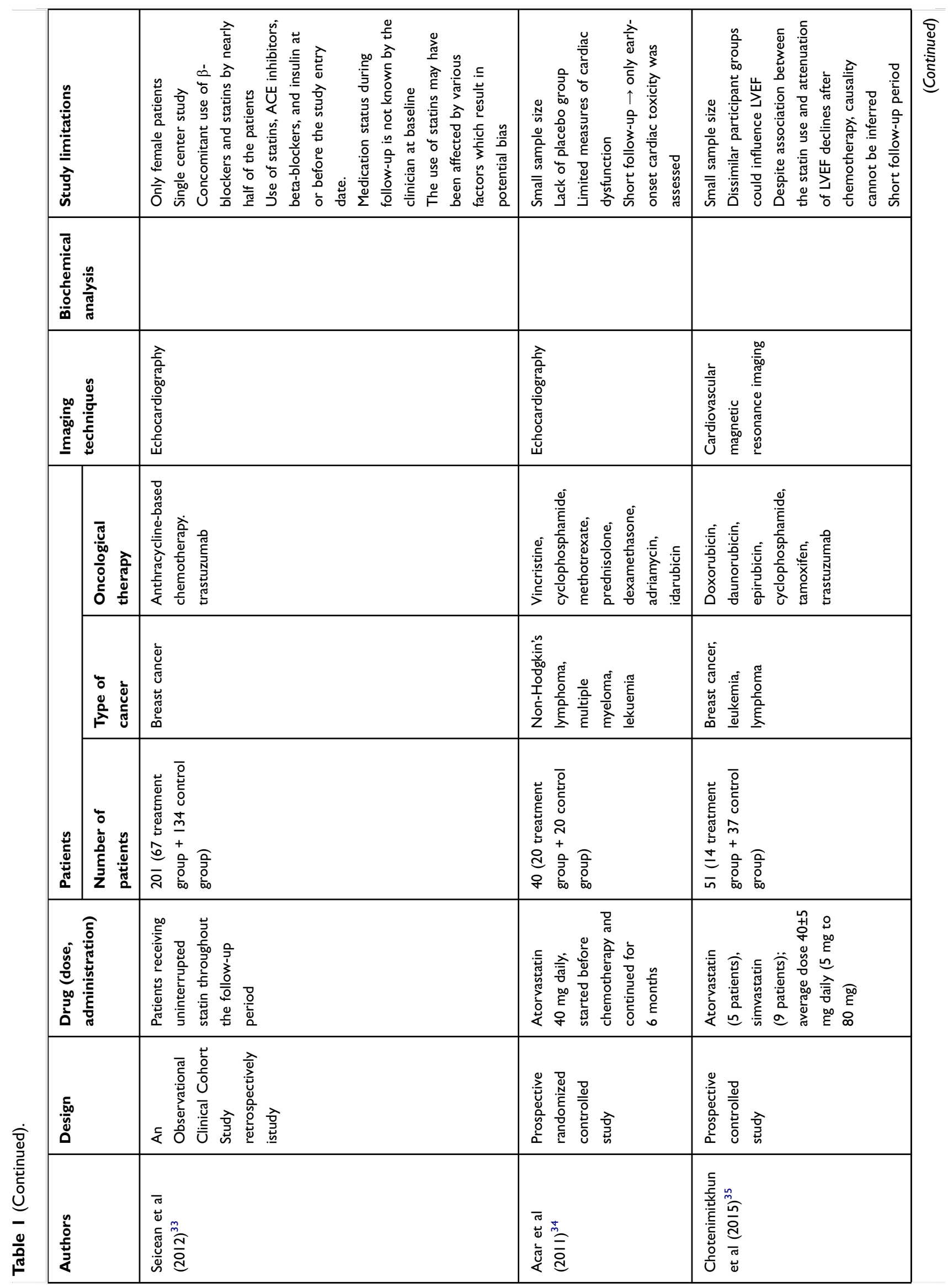




\begin{tabular}{|c|c|c|}
\hline & 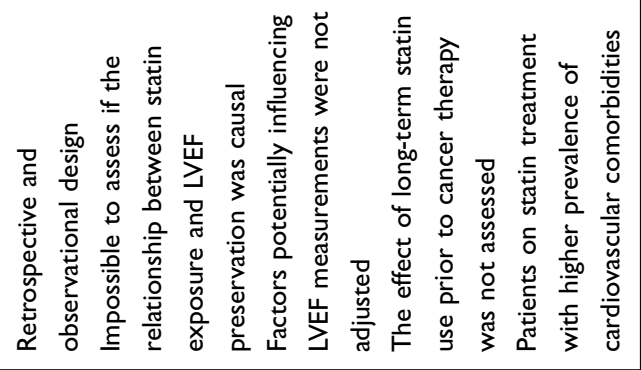 & 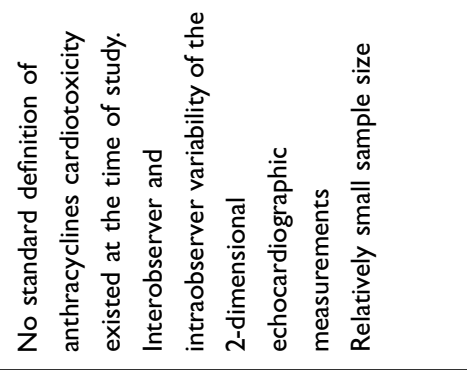 \\
\hline 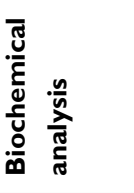 & & \\
\hline 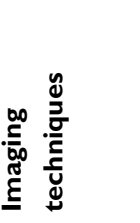 & 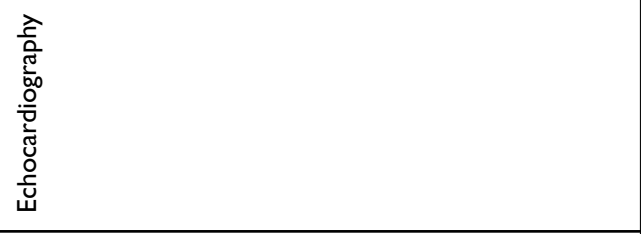 & 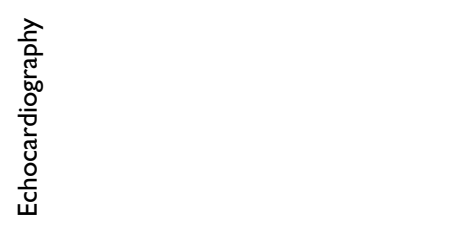 \\
\hline 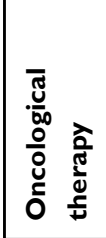 & 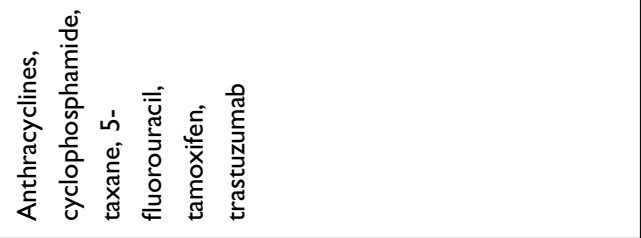 & 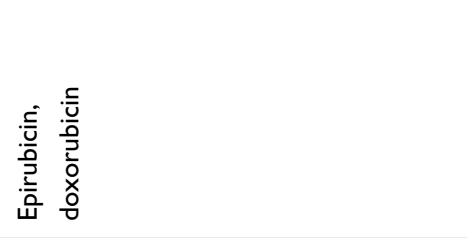 \\
\hline 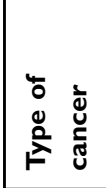 & 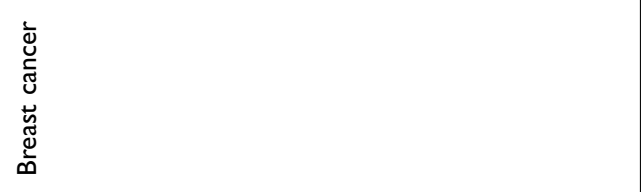 & 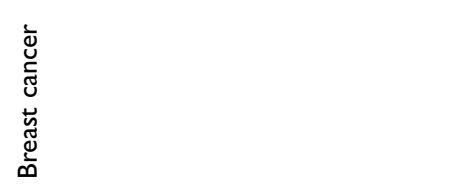 \\
\hline 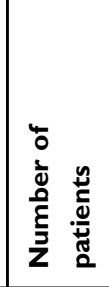 & 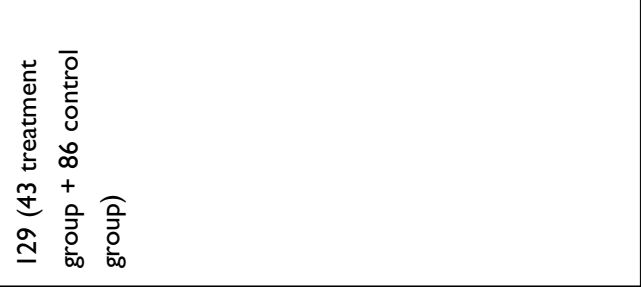 & 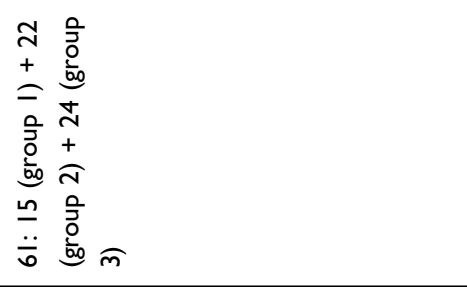 \\
\hline 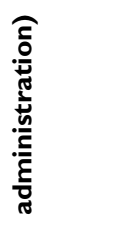 & 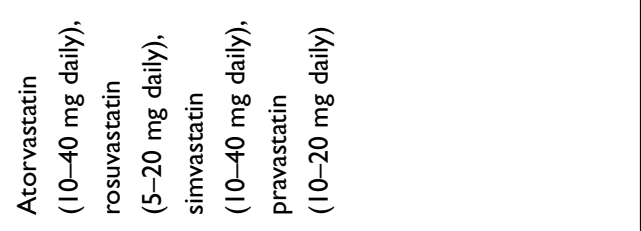 & 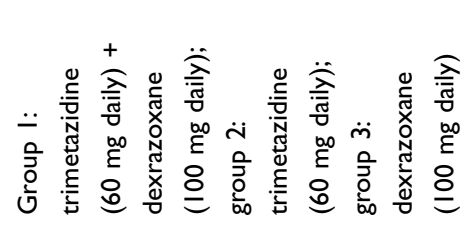 \\
\hline & 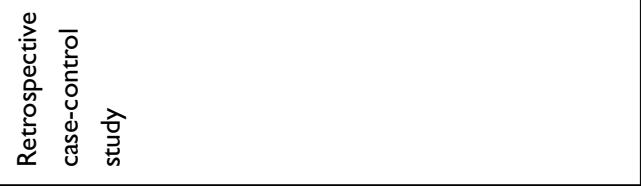 & 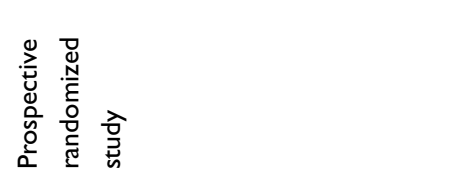 \\
\hline & 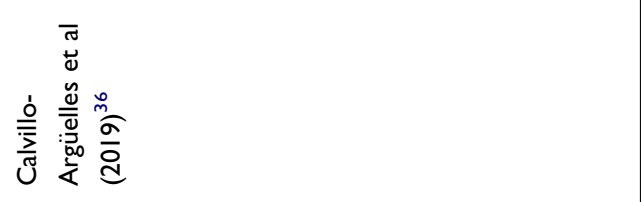 & 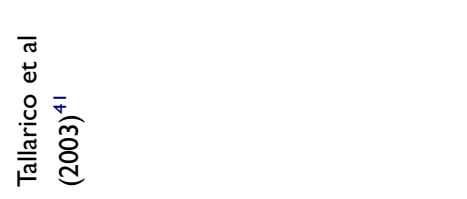 \\
\hline
\end{tabular}


frequency of heart failure in the group receiving metoprolol treatment. However, observed difference was not statistically significant. ${ }^{22}$ Gulati et al (Table 1) have found in the PRADA study that $78 \pm 32 \mathrm{mg}$ daily metoprolol did not affect LVEF, ECV (extracellular volume) fraction, total $\mathrm{ECV}$, or total cellular volume in patients on anthracycline therapy. The therapy was safe, with no severe adverse reactions and no patients withdrew from the study. ${ }^{23,24}$

\section{Angiotensin-converting enzyme inhibitors}

\section{Enalapril}

Cardinale et al (Table 1) used enalapril (20 $\mathrm{mg} /$ day) in a group of 56 cancer patients receiving high-dose chemotherapy. The treatment was started one month after the last chemotherapy cycle and was continued for one year. The study revealed persistently high $\mathrm{TnI}$ increase, associated with a greater LVEF reduction, in the control group (58 patients who did not receive enalapril). Administration of enalapril prevented a reduction in LVEF and an increase in end-diastolic and end-systolic volumes; moreover, the drug was well tolerated in most patients. ${ }^{25}$ These results were the premise for conducting research during which the drug was administered simultaneously with other cardiovascular agents commonly recommended as initial treatment of heart failure. First such study was the OVERCOME trial (Table 1), carried out on 90 patients with leukemia or malignant hemopathies, which revealed that patients receiving enalapril and carvedilol had a lower incidence of death and heart failure after intensive chemotherapy; the treatment also protected from negative changes in LVEF. There was no interaction between the drugs concerning the effect on LVEF and troponin I (TnI) increase. The study also confirmed the safety of such treatment: only three patients discontinued enalapril, while two patients stopped carvedilol and one withdrew from taking both drugs. ${ }^{26}$

\section{Perindopril}

Perindopril was assessed in MANTICORE study (Table 1) the first randomized, placebo-controlled trial for the prevention of trastuzumab-mediated cardiotoxicity. The drug was titrated weekly to $8 \mathrm{mg}$ and attenuated trastuzumabmediated declines in LVEF. However, it did not prevent ventricular remodeling. No significant adverse effects were noted during the study; lower blood pressure was observed in post-chemotherapy assessment. ${ }^{27}$

\section{Angiotensin 2 receptor blockers}

\section{Candesartan}

According to the results of PRADA study (Table 1), candesartan (26 $\pm 9 \mathrm{mg}$ daily) did not reduce circulating TnI (increased during anthracycline therapy) showing that angiotensin receptor blockers (ARB) had no effect on direct anthracyclines cardiotoxicity. However, they can affect remodeling of the myocardium which takes place after cardiac injury: angiotensin II directly stimulates protein synthesis in myocytes, causes myocyte hypertrophy, and induces an increase in left ventricular mass independent of pressure overload. The researchers observed that patients receiving candesartan had significantly lower LVEF decline compared to the placebo group. The safety profile of the treatment was good, with no serious adverse effects and no patients withdrawn. ${ }^{28}$ The study has also confirmed the effect of candesartan on the dose-dependent anthracyclineinduced increase in myocardial ECV - it was found that concomitant treatment with candesartan led to a reduction of left ventricular total cellular volume. ${ }^{24}$ Additionally, Liu et al (Table 1) observed that low-dose carvedilol (from $2.5 \mathrm{mg}$ twice a day at first cycle to $5 \mathrm{mg}$ twice a day) combined with candesartan ( $2.5 \mathrm{mg}$ once a day) in patients on anthracycline therapy did not prevent LVEF decrease, however it resulted in protection from intracellular damage (only $10 \%$ of the group had significantly higher expression of troponin) and ECG abnormalities. ${ }^{29}$

On the other hand, Boekhout et al (Table 1) did not confirm the protective effect of candesartan ( $32 \mathrm{mg}$ /day) in patients with breast cancer receiving anthracycline-containing chemotherapy followed by trastuzumab. The study has also found a single nucleotide polymorphism (SNP) Ala1170Pro in ERBB2 gene associated with the probability of cardiotoxicity. ${ }^{30}$

\section{Telmisartan}

Cadeddu et al (Table 1) have found that $40 \mathrm{mg}$ daily telmisartan (started one week before chemotherapy) in patients taking epirubicin resulted in strain rate normalization and reduction in epirubicin-induced radical species: at $200 \mathrm{mg} / \mathrm{m}^{2}$ epirubicin, strain rate in both telmisartan and placebo groups was reduced, however the decrease observed in the placebo group was significantly greater. Moreover, after an increase in cumulative epirubicin dose, 
strain rate in the telmisartan group increased, almost reaching the baseline level. ${ }^{31}$ The long-term positive effect was confirmed in an 18-month follow-up study: the telmisartan group maintained the strain rate level, while a further decrease was observed in the placebo group. Moreover, the treatment protected the patients from an elevation of proinflammatory cytokines (interleukin-6: IL-6, tumor necrosis factor-alpha: TNF- $\alpha$ ) and reactive oxygen species (ROS). Although the drug was well tolerated, two patients developed significant hypotension which resulted in temporary reduction of telmisartan dose (from 40 to $20 \mathrm{mg}$ daily); after two weeks the full dose was restored. $^{28}$

\section{Aldosterone antagonists}

The renin-angiotensin-aldosterone system (RAAS) has a significant effect on remodeling the myocardium in postmyocardial damage. Therefore, aldosterone antagonists have been shown to protect cardiac muscle against anthracycline-induced cardiomyopathy by preserving LVEF, LVEDD, and LVESD. Akpek et al (Table 1) carried out a prospective, randomized, placebo-controlled, double-blind study on 83 patients with breast cancer, treated with an anthracycline (adriamycin, epirubicin) and spironolactone (25 mg/day) or placebo, confirming the changes in cardiac biomarkers and echocardiography (ECHO) parameters. TnI levels increased significantly in both groups during the treatment period but the much higher level was observed in the control group, while the serum NT-proBNP levels increased similarly in both studies groups. The decrease in LVEF in the control group was significantly higher than in the spironolactone group $(67.7 \pm 6.3$ vs $53.6 \pm 6.8 \mathrm{~mm})$. Spironolactone was found to provide significant protection of diastolic fraction. ${ }^{32}$ On the other hand, a recent experimental study on eplerenone did not confirm the protective effect in preventing doxorubicin-induced cardiotoxicity. ${ }^{12}$

\section{Statins}

Seicean et al (Table 1) studied women with breast cancer receiving anthracycline chemotherapy and found that statin use was associated with a lower risk of incident heart failure. However, the assessment of the effectiveness of statins was difficult because approximately $40 \%$ patients used other potentially cardioprotective medications (ACEIs and $\beta$-blockers) and had significantly less cardiovascular risk factors (family histories of cardiovascular disease and lower low-density lipoproteins). ${ }^{33}$ Cardioprotective effect of statins may be associated with their significant pleiotropic effects, such as antioxidative and anti-inflammatory properties. In the study of Acar et al (Table 1), atorvastatin was administered, in a dose $40 \mathrm{mg}$ daily for six months, to patients who had a history of chemotherapy or radiotherapy without cardiovascular diseases, treated with adriamycin or idarubicin. Atorvastatin has been shown to reduce a decrease in left ventricular ejection fraction $(p<0.0001)$ Moreover, the mean increase in LVEDD and LVESD was significantly lower in the statin arm, compared to the control group $\left(p=0.021 ; p<0.001\right.$, respectively). ${ }^{34}$ Similar results were demonstrated by Chotenimitkhun et al (Table 1) in the oncological participants with hyperlipidemia and other cardiovascular risk factors, who received atorvastatin or simvastatin. A high statin dose (40-80 mg/day) was associated with a nonsignificant increase in LVEF compared to the placebo group, in which LVEF declined significantly. ${ }^{35}$ Recent study by Calvillo-Argüelles et al has also confirmed cardioprotective effect in the group of women with breast cancer receiving trastuzumab-based therapy. ${ }^{36}$

\section{Ranolazine}

Cappetta et al performed an experimental study with ranolazine and suggested that this drug may protect cardiomyocytes from doxorubicin-induced oxidative stress. ${ }^{37}$ Other authors point out that the use of ranolazine may be beneficial in diastolic dysfunction and chemotherapeutic cardiotoxicity, due to its capacity to reduce late sodium current and cytosolic $\mathrm{Na}^{+}$, and consequently to counteract intracellular $\mathrm{Ca}^{2+}$ accumulation. On the other hand, concomitant use of ranolazine and doxorubicin can be limited because of potential interaction, associated with absorption and biotransformation. ${ }^{38-40}$

\section{Trimetazidine}

Cardioprotective properties of trimetazidine $(60 \mathrm{mg} /$ day $)$ were assessed in 61 patients with breast cancer treated with an anthracycline (Table 1). The results showed that trimetazidine, similarly to dexrazoxane, provided a cardioprotective effect. Its efficacy was associated with protection against subacute and chronic subclinical cardiotoxicity with no significant changes in diastolic function after one year of follow-up. ${ }^{41}$

\section{Ivabradine}

So far, there are no clinical trials concerning ivabradine as a cardioprotective agent for oncological patients, despite its hemodynamic and biochemical parameters. A case report, published by de Gregorio et al, reported full 
restoration of left ventricular function, with no residual myocardial damage, after applying combination therapy with ivabradine, lisinopril and multivitamin supplementation. This finding supports the need for an experimental study on the potential therapeutic effect of ivabradine against doxorubicin-induced cardiotoxicity. ${ }^{42,43}$

\section{Coenzyme Q10}

First studies showing the potential cardioprotective effect of coenzyme Q10 (CoQ10) were published in the previous century. ${ }^{44-46}$ Recently, Mustafa et al performed an experimental study on rats treated orally with a single dose of doxorubicin $(10 \mathrm{mg} / \mathrm{kg})$ - they observed that CoQ10 supplementation $(200 \mathrm{mg} / \mathrm{kg})$ improved the functional and structural integrity of the myocardium. ${ }^{47}$

\section{Pharmacogenetics}

Genetic diversity can influence the functioning of various drugs. It may also affect the risk of developing druginduced cardiotoxicity. The progress in pharmacogenetics can, therefore, increase the possibilities of avoiding this adverse effect. According to Canadian Pharmacogenomics Network for Drug Safety Clinical Practice Recommendations Group, three SNPs in three genes have been found to be associated with increased risk of anthracycline cardiotoxicity in pediatric population: RARG rs2229774 (retinoic acid receptor gamma), SLC28A3 rs7853758 (solute carrier family 28 member 3) and UGT1A6*4 (UDP glucuronosyltransferase family 1 member a6) rs17863783. Assessment of these polymorphisms was therefore recommended for pediatric patients undergoing doxorubicin or daunorubicin treatment. No such recommendation was eligible for adult patients due to the lack of studies on adult populations. One of such studies was recently published by Schneider et al who found rs28714259 polymorphism associated with increased risk of anthracycline cardiotoxicity. However, further studies are required to provide recommendations for the adult population. ${ }^{48,49}$

\section{Summary}

Despite the large and an increasing scale of the problem of clinical oncological treatment in the form of cardiotoxicity, data from clinical trials carried out so far do not allow for the creation of clear recommendations regarding the use of cardiovascular drugs in the prevention of this complication. This is due to heterogeneous and small-sized populations of patients treated with various antitumor drugs that are cardiotoxic in different dosing regimens. Additionally, the cardioprotective drugs used in clinical trials were administered in a wide range of doses. The main purpose of their application was to assess their effectiveness in the prevention of primary development of post-anthracycline cardiomyopathy and heart failure.

The most serious cardiological adverse effect of oncological treatment (epidemiology, clinical consequences) is cardiomyopathy, which may lead to heart failure. Current data suggests the usefulness in cardioprotection of only some cardiovascular drugs recommended for the treatment of heart failure. The most promising examples from the review include carvedilol and nebivolol, enalapril, candesartan and the combination of carvedilol and enalapril. Both $\beta$-blockers and RAA inhibitors prevent adverse remodeling, and their combination improves prognosis and reduces mortality. The advantage of $\beta$-blockers is that they correct the elevated heart rate (resulting from autonomic disorders caused by anthracyclines), which is an independent factor of morbidity and mortality. These cardiovascular drugs not only are effective in the prevention of left ventricular dysfunction, but also in other forms of cardiotoxicity (hypertension, arrhythmia, myocardial ischemia). Their use in patients without cardiovascular disease is not associated with the risk of serious adverse effects, especially if their dosage is gradual. Alternatively, in case of adverse reactions, statins might be considered. Besides, an important element of cardioprotection strategy in these patients should be the modification of lifestyle (eg, smoking, alcohol consumption, low physical activity) which can have a negative effect on cardioprotective drug efficacy.

Currently, the lack of recommendations concerning cardioprotection in oncological patients increases the risk of cardiotoxicity which may lead to serious consequences for health and life. The "2016 European Guidelines on cardiovascular disease prevention in clinical practice" have listed $\beta$-blockers, ACEIs, dexrazoxane, and statins as prophylactic agents to reduce chemotherapy-induced cardiotoxicity. Based on the clinical studies discussed in our review, the guidelines' suggestions may be expanded/enhanced with:

1. introducing cardioprotective agents only in patients with subclinical heart injury (confirmed by increased troponin, decrease in global longitudinal strain: GLS, reduction of LVEF)

2. titrating the dose of the cardiovascular drug, starting from the lower dose and reaching the most optimal 
dose for the patient; adverse drug reactions monitoring must be mandatory

3. monitoring myocardial damage with imaging technology (eg, ECHO, nuclear magnetic resonance, equilibrium radionuclide angiocardiography/multigated acquisition) and troponin level

The above suggestions relate to the primary prevention of chronic cardiotoxicity. Their effectiveness seems to us to be dependent on strict adherence to the principles of detecting and monitoring cardiotoxicity. Ongoing advance in cardiovascular diagnostics (biochemical markers, imaging technologies) gives tools to define new targets for assessing cardioprotection efficacy (NT-proBNP, troponin, GLS besides ejection fraction).

\section{Disclosure}

The authors report no conflicts of interest in this work.

\section{References}

1. World Health Oganization [homepage on the Internet]. Geneva: The top 10 causes of death; 2018. Available from: http://www.who.int/news-room/ fact-sheets/detail/the-top-10-causes-of-death. Accessed 04 January 2019

2. Araujo-Gutierrez R, Ibarra-Cortez SH, Estep JD, et al. Incidence and outcomes of cancer treatment-related cardiomyopathy among referrals for advanced heart failure. Cardio-Oncology. 2018;4:3. doi:10.1186/ s40959-018-0029-y

3. Chang HM, Moudgil R, Scarabelli T, Okwuosa TM, Yeh ETH. Cardiovascular complications of cancer therapy: best practices in diagnosis, prevention, and management: part 1. J Am Coll Cardiol. 2017;70:2536-2551. doi:10.1016/j.jacc.2017.09.1096

4. Oliveira GH, Qattan MY, Al-Kindi S, Park SJ. Advanced heart failure therapies for patients with chemotherapy-induced cardiomyopathy. Circ Heart Fail. 2014;7(6):1050-1058. doi:10.1161/CIRCHEART FAILURE.114.001292

5. Menna P, Salvatorelli E. Primary prevention strategies for anthracycline cardiotoxicity: a brief overview. Chemotherapy. 2017;62:159168. doi: $10.1159 / 000455823$

6. European Medicines Agency [homepage on the Internet]. Amsterdam: Assessment report. Referral under Article 13 of Regulation (EC) No 1234/2008. Cardioxane; 2017. Available from: http://www.ema europa.eu/docs/en_GB/document_library/Referrals_document/ Cardioxane_13/WC500232245.pdf Accessed 04 January 2019

7. Korzeniowska K, Jankowski J, Cieślewicz A, Jabłecka A. Current approach for detection of sub-clinical left ventricular dysfunction associated with chemotherapy. Pharmacol Rep. 2015;67:1098-1102. doi:10.1016/j.pharep.2015.03.010

8. Curigliano G, Cardinale D, Suter T, et al.; on behalf of the ESMO Guidelines Working Group. Cardiovascular toxicity induced by chemotherapy, targeted agents and radiotherapy. ESMO Clin Pract Guidelines Ann Oncol. 2012;7:155-166.

9. Zamorano JL, Lancellotti P, Rodriguez Muñoz, et al. 2016 ESC position paper on cancer treatments and cardiovascular toxicity developed under the auspices of the ESC Committee for Practice Guidelines The Task Force for cancer treatments and cardiovascular toxicity of the European Society of Cardiology (ESC). Eur Heart J. 2016;37:27682801. doi:10.1093/eurheartj/ehw211
10. Piepoli MF, Hoes AW, Agewall S, et al. European guidelines on cardiovascular disease prevention in clinical practice: the Sixth Joint Task Force of the European Society of Cardiology and Other Societies on Cardiovascular Disease Prevention in Clinical Practice (constituted by representatives of 10 societies and by invited experts) developed with the special contribution of the European Association for Cardiovascular Prevention \& Rehabilitation (EACPR). Eur Heart J. 2016;2016(37):2315-2381.

11. Salvatorelli E, Menna P, Cantalupo E, et al. The concomitant management of cancer therapy and cardiac therapy. Biochim Biophys Acta. 2015;1848:2727-2737. doi:10.1016/j.bbamem.2015.01.003

12. Hullin R, Métrich M, Sarre A, et al. Diverging effects of enalapril or eplerenone in primary prevention against doxorubicin-induced cardiotoxicity. Cardiovasc Res. 2018;114:272-281. doi:10.1093/cvr/cvx162

13. Nakamae H, Tsumura K, Terada Y, et al. Notable effects of angiotensin II receptor blocker, valsartan, on acute cardiotoxic changes after standard chemotherapy with cyclophosphamide, doxorubicin, vincristine, and prednisolone. Cancer. 2005;104:2492-2498. doi: $10.1002 /$ cncr. 21478

14. Imbaby S, Ewais M, Essawy S, Farag N. Cardioprotective effects of curcumin and nebivolol against doxorubicin-induced cardiac toxicity in rats. Hum Exp Toxicol. 2014;33(8):800-813. doi:10.1177/ 0960327114527628

15. Kaya MG, Ozkan M, Gunebakmaz O, et al. Protective effects of nebivolol against anthracycline-induced cardiomyopathy: a randomized control study. Int $J$ Cardiol. 2013;167:2306-2310. doi:10.1016/j.ijcard.2012.06.023

16. Kalay N, Basar E, Ozdogru I, et al. Protective effects of carvedilol against anthracycline-induced cardiomyopathy. $\mathrm{J} \mathrm{Am} \mathrm{Coll} \mathrm{Cardiol.}$ 2006;48:2258-2262. doi:10.1016/j.jacc.2006.07.052

17. Elitok A, Oz F, Cizgici AY, et al. Effect of carvedilol on silent anthracycline-induced cardiotoxicity assessed by strain imaging: a prospective randomized controlled study with six-month follow-up. Cardiol J. 2014;21:509-515. doi:10.5603/CJ.a2013.0150

18. Zamani B, Salehi R, Esfahani A. Protective effect of carvedilol against anthracycline-induced cardiomyopathy on patients with breast cancer and lymphoma. Int J Adv Med. 2018;5:16-20. doi:10.18203/ 2349-3933.ijam20180061

19. Avila MS, Ayub-Ferreira SM, de Barros Wanderley MR Jr, et al. Carvedilol for prevention of chemotherapy-related cardiotoxicity: the CECCY trial. J Am Coll Cardiol. 2018;71:2281-2290. doi:10.1016/j. jacc.2018.02.049

20. Kheiri B, Abdalla A, Osman M, et al. Meta-analysis of carvedilol for the prevention of anthracycline-induced cardiotoxicity. Am J Cardiol. 2018;122:1959-1964. doi:10.1016/j.amjcard.2018.08.039

21. Pituskin E, Mackey JR, Koshman S, et al. Multidisciplinary approach to novel therapies in cardio-oncology research (MANTICORE 101-breast): a randomized trial for the prevention of trastuzumab-associated cardiotoxicity. J Clin Oncol. 2017;35:870-877. doi:10.1200/JCO.2016.68.7830

22. Georgakopoulos P, Roussou P, Matsakas E, et al. Cardioprotective effect of metoprolol and enalapril in doxorubicin-treated lymphoma patients: a prospective, parallel-group, randomized, controlled study with 36-month follow-up. Am J Hematol. 2010;85:894-896. doi:10.1002/ajh.21840

23. Gulati G, Heck SL, Ree AH, et al. Prevention of cardiac dysfunction during adjuvant breast cancer therapy (PRADA): a $2 \times 2$ factorial, randomized, placebo-controlled, double-blind clinical trial of candesartan and metoprolol. Eur Heart J. 2016;37:1671-1680. doi:10.10 93/eurheartj/ehw022

24. Heck SL, Gulati G, Hoffmann P, et al. Effect of candesartan and metoprolol on myocardial tissue composition during anthracycline treatment: the PRADA trial. Eur Heart J Cardiovasc Imaging. 2018;19:544-552. doi:10.1093/ehjci/jex159

25. Cardinale D, Colombo A, Sandri MT, et al. Prevention of high-dose chemotherapy-induced cardiotoxicity in high-risk patients by angiotensin-converting enzyme inhibition. Circulation. 2006;114:24742481. doi:10.1161/CIRCULATIONAHA.106.635144 
26. Bosch X, Rovira M, Sitges M, et al. Enalapril and carvedilol for preventing chemotherapy-induced left entricular systolic dysfunction in patients with malignant hemopathies: the OVERCOME trial (preventiOn of left Ventricular dysfunction with enalapril and caRvedilol in patients submitted to intensive ChemOtherapy for the treatment of Malignant hEmopathies). $J$ Am Coll Cardiol. 2013;61:2355-2362.

27. Schwarz ER, Kersting PH, Reffelmann T, et al. Cardioprotection by carvedilol: antiapoptosis is independent of beta-adrenoceptor blockage in the rat heart. J Cardiovasc Pharmacol Ther. 2003;8:207-215. doi:10.1177/107424840300800306

28. Dessì M, Madeddu C, Piras A, et al. Long-term, up to 18 months, protective effects of the angiotensin II receptor blocker telmisartan on Epirubin-induced inflammation and oxidative stress assessed by serial strain rate. Springerplus. 2013;2:198. doi:10.1186/2193-18012-198

29. Liu L, Liu ZZ, Liu YY, et al. Preventive effect of low-dose carvedilol combined with candesartan on the cardiotoxicity of anthracycline drugs in the adjuvant chemotherapy of breast cancer. Zhonghua Zhong Liu Za Zhi. 2013;35:936-940.

30. Boekhout AH, Gietema JA, Milojkovic Kerklaan B, et al. Angiotensin II-receptor inhibition with candesartan to prevent trastuzumab-related cardiotoxic effects in patients with early breast cancer. A randomized clinical Trial. JAMA Oncol. 2016;2:1030-1037. doi:10.1001/jamaoncol.2016.1726

31. Cadeddu C, Piras A, Mantovani G, et al. Protective effects of the angiotensin II receptor blocker telmisartan on epirubicin-induced inflammation, oxidative stress, and early ventricular impairment. Am Heart J. 2010;160:487.e1-487. doi:10.1016/j.ahj.2010.05.037

32. Akpek M, Ozdogru I, Sahin O, et al. Protective effects of spironolactone against anthracycline-induced cardiomyopathy. Eur $J$ Heart Fail. 2015;17:81-89. doi:10.1002/ejhf.196

33. Seicean S, Seicean A, Plana JC, Budd GT, Marwick TH. Effect of statin therapy on the risk for incident heart failure in patients with breast cancer receiving anthracycline chemotherapy: an observational clinical cohort study. J Am Coll Cardiol. 2012;60:2384-2390. doi:10.1016/j.jacc.2012.07.067

34. Acar Z, Kale A, Turgut M, et al. Efficiency of ATORVASTATIN in the protection of anthracycline-induced cardiomyopathy. J Am Coll Cardiol. 2011;9:988-989. doi:10.1016/j.jacc.2011.05.025

35. Chotenimitkhun R, D’Agostino R Jr, Lawrence JA, et al. Chronic statin administration may attenuate early anthracycline-associated declines in left ventricular ejection function. Can J Cardiol. 2015;31:302-307. doi:10.1016/j.cjca.2014.11.020

36. Calvillo-Argüelles O, Abdel-Qadir H, Michalowska $\mathrm{M}$, et al. Cardioprotective effect of statins in patients with her2-positive breast cancer receiving trastuzumab therapy. Can J Cardiol. 2019;35:153159. doi:10.1016/j.cjca.2018.11.028
37. Cappetta D, Esposito G, Coppini R, et al. Effects of ranolazine in a model of doxorubicin-induced left ventricle diastolic dysfunction. $\mathrm{Br}$ J Pharmacol. 2017;174:3696-3712. doi:10.1111/bph.13791

38. Corradi F, Paolini L, De Caterina R. Ranolazine in the prevention of anthracycline cardiotoxicity. Pharmacol Res. 2014;79:88-102. doi:10.1016/j.phrs.2013.11.001

39. Mihos CG, Krishna RK, Kherada N, Larrauri-Reyes M, Tolentino A, Santana O. The use of ranolazine in non-angina cardiovascular disorders: a review of current data and ongoing randomized clinical trials. Pharmacol Res. 2016;103:49-55. doi:10.1016/j.phrs.2015.10.018

40. Minotti G. Pharmacology at work for cardio-oncology: ranolazine to treat early cardiotoxicity induced by antitumor drugs. J Pharmacol Exp Ther. 2013;346:343-349. doi:10.1124/jpet.113.204057

41. Tallarico D, Rizzo V, Di Maio F, et al. Myocardial cytoprotection by trimetazidine against anthracycline-induced cardiotoxicity in anticancer chemotherapy. Angiology. 2003;54:219-227. doi:10.1177/ 000331970305400212

42. de Gregorio C, Potenza G, Ferraro G. Effectiveness of the combination therapy with lisinopril, ivabradine and multivitamin supplementation in anthracycline-induced severe cardiotoxicity. Int J Cardiol. 2014;176:1374-1376. doi:10.1016/j.ijcard.2014.07.272

43. Colak MC, Parlakpinar H, Tasdemir S, et al. Therapeutic effects of ivabradine on hemodynamic parameters and cardiotoxicity induced by doxorubicin treatment in rat. Hum Exp Toxicol. 2012;31:945-954. doi:10.1177/0960327112438288

44. Okuma K, Furuta I, Ota K. Protective effect of coenzyme Q10 in cardiotoxicity induced by Adriamycin. Gan To Kagaku Ryoho. 1984;11:502-508.

45. Tsubaki K, Horiuchi A, Kitani T, et al. Investigation of the preventive effect of CoQ10 against the side-effects of anthracycline antineoplastic agents. Gan To Kagaku Ryoho. 1984;11:1420-1427.

46. Iarussi D, Auricchio U, Agretto A, et al. Protective effect of coenzyme Q10 on anthracyclines cardiotoxicity: control study in children with acute lymphoblastic leukemia and non-Hodgkin lymphoma. Mol Aspects Med. 1994;15:207-212. doi:10.1016/ 0098-2997(94)90030-2

47. Mustafa HN, Hegazy GA, Awdan SAE, AbdelBaset M. Protective role of CoQ10 or L-carnitine on the integrity of the myocardium in doxorubicin induced toxicity. Tissue Cell. 2017;49:410-426. doi:10.1016/j.tice.2017.03.007

48. Chang VY, Wang JJ. Pharmacogenetics of chemotherapy-induced cardiotoxicity. Curr Oncol Rep. 2018;20:52. doi:10.1007/s11912018-0696-8

49. Schneider BP, Shen F, Gardner L, et al. Genome-Wide Association Study for anthracycline-induced congestive heart failure. Clin Cancer Res. 2017;23:43-51. doi:10.1158/1078-0432.CCR-16-0908
Therapeutics and Clinical Risk Management

\section{Publish your work in this journal}

Therapeutics and Clinical Risk Management is an international, peerreviewed journal of clinical therapeutics and risk management, focusing on concise rapid reporting of clinical studies in all therapeutic areas, outcomes, safety, and programs for the effective, safe, and sustained use of medicines. This journal is indexed on PubMed Central, CAS,
EMBase, Scopus and the Elsevier Bibliographic databases. The manuscript management system is completely online and includes a very quick and fair peer-review system, which is all easy to use. Visit http://www.dovepress.com/testimonials.php to read real quotes from published authors. 